\title{
Archivos

\section{Declaración sobre la correspondencia entre expresiones de lenguaje y tipo de evidencia utilizada en la descripción de datos de resultados de estudios}

\section{Statement on matching language to the type of evidence used in describing outcomes data}

\section{Editores de revistas del grupo HEART}

Recibido el 2 de abril de 2013; aceptado el 26 de abril de 2013

Hay muchos tipos de estudios que pueden llevarse a cabo para obtener una evidencia en la investigación clínica. Entre ellos se encuentran los análisis observacionales retrospectivos, los estudios de casos y controles, y los ensayos controlados y aleatorizados. Cada uno de estos tipos de análisis tiene puntos fuertes y débiles, pero lo más importante es que cada uno aporta un tipo de conclusiones sobre una intervención que difiere del que proporcionan los demás.

Tal como pone de relieve una serie de ejemplos presentados en una revisión publicada por separado ${ }^{1}$, la elección inadecuada de las palabras para describir los resultados puede conducir a inexactitudes científicas. En consecuencia, los editores del grupo HEART (representantes de revistas cardiovasculares de todo el mundo) recomiendan que todos los investigadores y editores elijan cuidadosamente las expresiones del lenguaje que se «correspondan» con el tipo de estudio realizado, sin ir más allá de lo que indican los datos ni extraer conclusiones erróneas acerca de la causalidad cuando no es posible establecerlas.

Para ilustrarlo con un ejemplo, cuando se presentan los resultados de un estudio observacional que muestra un número de muertes inferior en un grupo en comparación con otro, deben usarse expresiones descriptivas, como

\footnotetext{
Documento publicado anteriormente en Rev Esp Cardiol. 2013;66:155.
}

Correo electrónico: susana.baez.acm@gmail.com
Tabla 1 Expresiones de lenguaje sugeridas según el tipo de estudio

Ensayo con
distribución
aleatoria

Tipo de expresiones

Expresio- «Redujo el riesgo «Se observó un riesgo

nes en»

descripti-

vas

Nombres «Reducción del

descripti- riesgo relativo»,

vos

«beneficio»

inferior», «hay una

relación», «hay una asociación»

«Diferencia de riesgo», «cociente/razón de riesgos»

Verbos

«Afectó», «causó», «moduló el

riesgo», «el tratamiento dio lugar a», «redujo el riesgo»

Términos incorrectos/evitar el uso
«Correlacionado con», «se asocia a»
«Redujo el riesgo» (verbo en voz activa), «disminuyó el riesgo» (verbo en voz activa), «produjo un beneficio»

Reproducida con permiso de Kohli y Cannon ${ }^{1}$. 
«la intervención se asocia a una mortalidad inferior» y no expresiones de carácter definitivo como «la intervención reduce la mortalidad». En cambio, al presentar los resultados de un ensayo controlado y aleatorizado realizado de manera rigurosa con un seguimiento completo, en el que la única diferencia detectada entre los 2 grupos ha sido la intervención, puede ser apropiado utilizar expresiones algo más taxativas como «la intervención redujo el riesgo». En la tabla 1 se indican algunos otros ejemplos de expresiones de lenguaje aplicables a distintos tipos de estudio.

En conclusión, todos los artículos deben ser redactados y corregidos no solo en lo relativo a su exactitud científica, sino también en cuanto al uso de un lenguaje apropiado al describir el nivel de evidencia que aporta el estudio.

\section{Conflicto de intereses}

Los autores declaran no tener ningún conflicto de intereses.

\section{Bibliografía}

1. Kohli P, Cannon CP. The importance of matching language to type of evidence: avoiding the pitfalls of reporting outcomes data. Clin Cardiol. 2012;35:714-7. 